\title{
A THEOREM ON THE ZEROS OF ENTIRE FUNCTIONS OF EXPONENTIAL TYPE
}

\section{KRISHNAMURTHY}

1. Introduction. In a recent paper [2, p. 417] Rubel proves the following

THEOREM A. Let $f(z)$ be an entire function satisfying

$$
\begin{array}{rrr}
f(z) & =O(1) \exp (\gamma|z|) & \text { for all } z, \\
f(i y) & =O(1) \exp (c|y|) & \text { for some } c<\pi, \\
f\left(a_{n}\right) & =0 & \text { for each } a_{n} \text { in } A, \text { where } A \text { is }
\end{array}
$$

a set of positive integers with upper density $\Delta$. Then, in order that $f(z)$ may vanish identically, the necessary and sufficient condition is $\Delta=1$.

It is natural to inquire "What may be the type on the imaginary axis when there are more lines of zeros in the right half plane with similar density conditions?" In this paper I extend the above theorem to the case when there are $N$ sequences of zeros of $f(z)$ lying on $N$ lines $\theta=\theta_{k},\left|\theta_{k}\right|<\pi / 2, k=1 \cdots N$. Let us write

$$
\beta_{k n}=r_{k n} e^{i \theta_{k}}, \quad n=1,2, \cdots
$$

where $r_{k n}$ is a positive integer. Let the upper density of the sequence of moduli on the line $\theta=\theta_{k}$ be $\Delta(k)$ defined by

$$
\Delta(k)=\limsup _{t \rightarrow \infty} \frac{A_{k}(t)}{t}
$$

where $A_{k}(t)$ is the number of terms of the sequence $\left(r_{k n}\right)$ less than or equal to $t$. The generalization of Theorem A can now be stated as

TheOREM B. Let $f(z)$ be an entire function satisfying

$$
\begin{array}{r}
f(z)=O(1) \exp (\gamma|z|) \quad \text { for all } z, \\
f(i y)=O(1) \exp (c|y|) \text { for some } c<\pi\left(\cos \theta_{1}+\cdots+\cos \theta_{N}\right)
\end{array}
$$

and

$$
f\left(\beta_{k n}\right)=0 \quad k=1, \cdots N, n=1, \cdots .
$$

Then $f(z)$ vanishes identically if and only if

$$
\Delta(k)=1 \text {, }
$$$$
k=1, \cdots, N \text {. }
$$

Received by the editors August 8, 1957 . 
2. Proof of the necessity of condition (7). The method is this. We assume that (7) is not true, in the sense that at least one of the upper densities $\Delta(k)$ is less than 1 . Then we prove the existence of a function $f(z)$ not identically equal to zero, but satisfying all the conditions (4), (5) and (6) of the hypothesis. Such a function is

$$
f(z)=\prod_{k=1}^{N}\left\{\prod_{n=1}^{\infty}\left(1-\frac{z^{2}}{\beta_{k n}^{2}}\right)\right\}
$$

which evidently satisfies (6). That it also satisfies (4) follows from Lindelof's Theorem $([1$, p. 27$])$. It only remains to estimate the type on the imaginary axis.

Writing

$$
\phi_{k}(z)=\prod_{n=1}^{\infty}\left\{1-\frac{z^{2}}{\beta_{k n}^{2}}\right\} \quad k=1, \cdots, N \text {, }
$$

we have

$$
\log \phi_{k}(i y)=\sum_{n=1}^{\infty} \log \left\{1+\frac{y^{2} e^{-2 i \theta_{k}}}{r_{k n}^{2}}\right\}
$$

but we know

$$
\sum_{n=1}^{\infty} \log \left(1-\frac{z^{2}}{a_{n}^{2}}\right)=\int_{0}^{\infty} \log \left(1-\frac{z^{2}}{t^{2}}\right) d(A(t)),
$$

where $A(t)$ is the number of $a_{n}$ 's less than or equal to $t$. Integration by parts gives

$$
\sum_{n=1}^{\infty} \log \left(1-\frac{z^{2}}{a_{n}^{2}}\right)=-2 z^{2} \int_{0}^{\infty} \frac{A(t)}{t} \frac{d t}{\left(t^{2}-z^{2}\right)} .
$$

So (10) becomes

$$
\begin{aligned}
\log \phi_{k}(i y) & =2 y^{2} e^{-2 i \theta_{k}} \int_{0}^{\infty} \frac{A_{k}(t)}{t} \frac{d t}{\left(t^{2}+y^{2} e^{-2 i \theta_{k}}\right)} \\
& =2 y^{2} e^{-2 i \theta_{k}} \int_{0}^{\infty} \frac{A_{k}(t)}{t} \frac{\left(t^{2}+y^{2} \cos 2 \theta_{k}\right)+i y^{2} \sin 2 \theta_{k}}{\left.t^{4}+y^{4}+2 t^{2} y^{2} \cos 2 \theta_{k}\right)} d t .
\end{aligned}
$$

Taking real parts, we get

$$
\begin{aligned}
\log \left|\phi_{k}(i y)\right|= & 2 y^{4} \int_{0}^{\infty} \frac{A_{k}(t)}{t} \cdot \frac{d t}{\left(t^{4}+y^{4}+2 t^{2} y^{2} \cos 2 \theta_{k}\right)} \\
& +2 y^{2} \cos 2 \theta_{k} \int_{0}^{\infty} \frac{A_{k}(t)}{t} \cdot \frac{t^{2} d t}{\left(t^{4}+y^{4}+2 t^{2} y^{2} \cos 2 \theta_{k}\right)}
\end{aligned}
$$


Replacing $t$ by $y v$ and writing $A_{k}(y v) \leqq \Delta(k) \cdot y v+\epsilon$ for large $y$, we obtain

$$
\begin{aligned}
\log \left|\phi_{k}(i y)\right| \leqq & 2 y \Delta(k) \int_{0}^{\infty} \frac{d v}{\left(1+v^{4}+2 v^{2} \cos 2 \theta_{k}\right)} \\
& +2 y \Delta(k) \cos 2 \theta_{k} \int_{0}^{\infty} \frac{v^{2} d v}{\left(1+v^{4}+2 v^{2} \cos 2 \theta_{k}\right)} \\
& +O(1) \\
= & 2 y \Delta(k) \frac{\pi}{4 \cos \theta_{k}}+2 y \cos 2 \theta_{k} \Delta(k) \frac{\pi}{4 \cos \theta_{k}}+O(1) \\
= & \pi y \Delta(k) \cos \theta_{k}+O(1) .
\end{aligned}
$$

Hence,

$$
\log |f(i y)| \leqq \pi y \sum_{k=1}^{N} \Delta(k) \cos \theta_{k}+O(1),
$$

which means

$$
c \leqq \pi \sum_{k=1}^{N} \Delta(k) \cos \theta_{k} .
$$

Our assumption that at least one of the densities $\Delta(k)$ is less than 1 now gives

$$
c<\pi \sum_{k=1}^{N} \cos \theta_{k}
$$

The function thus satisfies (5) and the proof of the "necessity" part of the theorem is complete.

3. Proof of the sufficiency of condition (7). Here we use a method quite similar to that used by Rubel ([2]) in proving Theorem A and consequently the working is almost the same.

In order to prove that ( 7$)$ is a sufficient condition we prove that

$$
L(k)=1, \quad k=1, \cdots, N
$$

is sufficient and then the earlier one will follow from the theorem that $\Delta(k)=1$ if and only if $L(k)=1$, for sets of positive integers [2, p. 418, Theorem 4]. Here $L(k)$ is the logarithmic block density of the set of positive integers $r_{k n}$ defined by

$$
L(k)=\inf _{\lambda>1} \limsup _{R \rightarrow \infty} \frac{1}{\log \lambda}\left\{\sum_{R \leqq r_{k n} \leqq \lambda R}\left(\frac{1}{r_{k n}}\right)\right\} .
$$

It is known $[2$, p. 424$]$ that generally $L(k) \leqq \Delta(k)$. 
We assume that (11) is true and then prove that any entire function satisfying (4), (5) and (6) ought to vanish identically. Suppose, on the other hand, there exists a function $f(z)$ satisfying (4), (5) and (6) but which is not identically zero. Let the zeros of this function in the right half plane be $z_{n}=a_{n} e^{i \phi_{n}}$. Then by Carleman's theorem [1, p. 2], we have, for any positive number $R$,

$$
\begin{aligned}
\sum_{a_{n} \leqq R}\left(\frac{1}{a_{n}}-\frac{a_{n}}{R^{2}}\right) \cos \phi_{n} & =\frac{1}{\pi R} \int_{-\pi / 2}^{\pi / 2} \log \left|f\left(R e^{i \theta}\right)\right| \cos \theta d \theta \\
+ & \frac{1}{2 \pi} \int_{0}^{R}\left(\frac{1}{y^{2}}-\frac{1}{R^{2}}\right) \log |f(i y) f(-i y)| d y+O(1) .
\end{aligned}
$$

We replace $R$ by $\lambda R$, subtract the above from the result thus obtained and then, discarding the (positive) terms on the Left side not involving the zeros $\left(\beta_{k n}\right)$, we obtain

$$
\begin{aligned}
\sum_{k=1}^{N}\left\{\sum _ { R < r _ { k n } \leqq \lambda R } \left(\frac{1}{r_{k n}}\right.\right. & \left.\left.\cos \theta_{k}\right)\right\}+\sum_{k=1}^{N}\left\{\sum_{r_{k n} \leqq R}\left(\frac{r_{k n}}{R^{2}} \cos \theta_{k}\right)\right\} \\
\leqq & \sum_{a_{n} \leqq \lambda R}\left(\frac{a_{n}}{\lambda^{2} R^{2}}\right)+\frac{1}{\pi \lambda R} \int_{-\pi / 2}^{\pi / 2} \log \left|f\left(\lambda R e^{i \theta}\right)\right| \cos \theta d \theta \\
& -\frac{1}{\pi R} \int_{-\pi / 2}^{\pi / 2} \log \left|f\left(R e^{i \theta}\right)\right| \cos \theta d \theta \\
& +\frac{1}{2 \pi} \int_{R}^{\lambda R}\left(\frac{1}{y^{2}}-\frac{1}{\lambda^{2} R^{2}}\right) \log |f(i y) f(-i y)| d y \\
& +\frac{1}{2 \pi} \int_{0}^{R}\left(\frac{1}{R^{2}}-\frac{1}{\lambda^{2} R^{2}}\right) \log |f(i y) f(-i y)| d y \\
& +O(1) .
\end{aligned}
$$

The estimates for all the terms on the Right side, based on the hypotheses (4) and (5) have been made by Rubel [2, pp. 419, 420 , (3.2), (3.5), (3.6), (3.7) and (3.8)] and, using them we finally obtain

$$
\sum_{k=1}^{N}\left\{\cos \theta_{k}\left(\sum_{R<r_{k n} \leqq \lambda R} \frac{1}{r_{k n}}\right)\right\} \leqq \frac{c}{\pi} \log \lambda+O(1),
$$

where the constants involved in the " $O$ " are independent of $\lambda$ and of $R$ for large values of $R$. Hence,

$$
\sum_{k=1}^{N}\left\{\cos \theta_{k} \cdot \limsup _{R \rightarrow \infty}\left(\sum_{R \leqq r_{k n} \leqq \lambda R} \frac{1}{r_{k n}}\right)\right\} \leqq \frac{c}{\pi} \log \lambda+O(1),
$$


which leads to

$$
\sum_{k=1}^{N} L(k) \cos \theta_{k} \leqq \frac{c}{\pi} .
$$

Our assumption of (11) now gives

$$
\sum_{k=1}^{N} \cos \theta_{k} \leqq \frac{c}{\pi} .
$$

This contradicts the hypothesis (5) and Theorem B is completely proved.

4. In the above, the hypothesis that $\left(r_{k n}\right)$ is a set of positive integers is used only in the sufficiency part of the proof. Relaxing this condition, we can assume that $\left(r_{k n}\right)$ is any set of positive real numbers satisfying the separation condition:

$$
r_{k, n+1}-r_{k, n} \geqq \alpha>0 .
$$

By arguments quite similar to the above, it is now easy to establish the following

THEOREM C. Let $f(z)$ be an entire function satisfying

$$
\begin{aligned}
& f(z)=O(1) \exp (\gamma|z|) \\
& \text { for all } z \text {, } \\
& f(i y)=O(1) \exp (c|y|) \text { for some } c<C\left(\cos \theta_{1}+\cdots+\cos \theta_{N}\right), \\
& f\left(\beta_{k n}\right)=0 \\
& \text { where }\left(\left|\beta_{k n}\right|\right) \text { satisfies (12). }
\end{aligned}
$$

Then, in order that $f(z)$ may vanish identically, (i) a necessary condition is

$$
\Delta(k) \geqq C / \pi \quad \text { for at least one } k,
$$

and (ii) a sufficient condition is

$$
L(k) \geqq C / \pi \quad \text { for all } k=1, \cdots, N .
$$

The conjecture of Rubel ([2, p. 422]) for the case $N=1, \theta_{1}=0$ is thus still open.

I finally thank Dr. V. Ganapathy Iyer for his guidance in the preparation of this paper.

\section{REFERENCES}

1. R. P. Boas, Entire functions, New York, Academic Press, 1954.

2. L. A. Rubel, Necessary and sufficient conditions for Carlson's theorem on entire functions, Trans. Amer. Math. Soc. vol. 83 (1956) pp. 417-429.

Annamalai University,

Annamalainagar, South India 